\title{
The Fetal Alcohol Spectrum Disorders: A Mini-Review
}

\author{
Michael AB Naafs* \\ Naafs International Health Consultancy, Europe
}

Received: January 01, 2018; Published: January 11, 2018

*Corresponding author: Michael AB Naafs, Internist-endocrinologist with a long clinical career in internal medicine and endocrinology, Naafs International Health Consultancy, Europe, Email: naafsmichael@gmail.com

\section{Abstract}

Since the first description of the fetal alcohol syndrome in 1973 the fetal alcohol spectrum disorders (FASDs) are still the leading preventable cause of birth defects,intellectual and neurodevelopmental disabilities. A recent WHO study estimates the global prevalence 7,7 per 1000 population,with large differences between countries.Renewed awareness results in new prevention and screening campaigning for this completely preventable global public health problem. In this mini-review, the various parts of FASD, as FAS, ARND and ARBD are discussed.

\section{Introduction}

The term fetal alcohol syndrome was first used in 1973 [1,2]. Fetal Alcohol spectrum disorders are caused by drinking alcoho during pregnancy $[3,4]$. Prenatal exposure to alcohol can damage the developing fetus and is the leading preventable cause of birth defects and intellectual and neurodevelopmental disabilities $[1,3,4]$. It presented as a cluster of birth defects[1]. Fetal alcohol spectrum disorders (FASDs) encompasses a range of possible diagnoses, including fetal alcohol syndrome (FAS), partial fetal alcohol syndrome (pFAS), alcohol-related neurodevelopmental disorder (ARND), alcohol related birth defects (ARBD) and neurobehavioral disorder associated with prenatal alcohol exposure (ND-PAE). FAS is a distinct clinical entity refering to a specific constellation of physical behavioral and cognitive abnormalities, resulting from prenatal alcohol exposure (PAE)-[1].

The lack of uniformly accepted diagnostic criteria for FAS and other related disorders has limited efforts to determine accurate prevalence figures. The World Health Organization (WHO) estimates the global prevalence to be 7,7 per 1000 population (95\% CI; 4,9-11,7 per 1000 population). The WHO European Region had the highest prevalence (19,8 per 1000 population) and the WHO Eastern Meditarranean Region had the lowest $(0,1$ per 1000 population).South Africa was estimated to have the highest prevalence of FASD at 111,1 per 1000 population, followed by Croatia (53,3/1000 population)-[5].

Alcohol-related birth defects and developmental disabilities are completely preventable,when pregnant women abstain from alcohol use.Neurocognitive and behavioral problems resulting from prenatal exposure are lifelong.Early recognition,diagnosis, and therapy along the FASD spectrum can result in improved outcomes. There is no amount of alcohol intake to consider safe.There is no safe trimester to drink alcohol. All forms of alcohol pose similar risk and binge drinking poses dose-related related risk to the developing fetus [6]. There is a global need to establish an universal public health message about the potential harm of prenatal alcohol exposure and a routine screening protocol,according to the WHO [5]. Globally, nearly 10 percent of women drink alcohol during pregnancy [7]. In this mini-review the various parts of the fetal alcohol spectrum disorders will be discussed. The pathogenesis, pathophysiology and treatment are beyond the scope of this mini-review.

\section{Fetal Alcohol Syndrome (FAS)}

For the diagnosis FAS, patients must have at least one growth abnormality eg short stature,as well as all three characterisic facial abnormalities;short palpebral fissure length,a thin upper lip and a smooth philtrum.They must also have at least one diagnosed structural or functional abnormality of the central nervous system, eg microcephaly or impaired executive function. Confirmation of intrauterine exposure to alcohol is not obligatory for the diagnosis [8].

Growth abnormalities in FAS: The percentiles for weight are significantly lower in children with FAS at birth and in following outpatient consultations,,compared with children without FAS in a retrospective study of 322 FAS children. Moreover 22\% had a body mass index below the third percentile compared with $3 \%$ of those withoit FAS[9]. Day et al. (cohort study,n=580) found, that 14 year old children,whose mothers had drunk alcohol in the first and second trimester of pregnancy showed reduced body weight, and maternal alcohol consumption in the first trimester led to smaller body length [10]. Explanation of the growth disturbance by other causes as eg prenatal deficiency states, hormonal disorders, malabsorption, malnutrition, neglect, and genetic syndromes should be excluded [11]. 
Facial anomalies in FAS: Several studies described the characteristic facial abnormalities in children with FAS [12-16]. Regardless of ethnicity and sex, the most powerful discriminating features for FAS proved to be smoothing of the philtrum, a thin upper lip and short palpabral length. These facial screening criteria for FAS showed a sensitivity of $100 \%$ and a specificity of $89,4 \%$. To add quantitative assessment of upper lip thickness and philtrum smoothness Astley and Clarren developed a lip-philtrum guide with five photographs comparable to a five-point Likert scale. Upper lip and philtrum scores of 4 or 5 are considered pathological in the context of suspected FAS $[17,18]$.

Central nervous system (CNS) abnormalities in FAS: Early injury of the brain,due to alcohol toxicity may be manifested by microcephaly. Affected children and adolescents show behavioral phenotypes of toxic damage to brain structures. As most studies are exploratory case-control studies no specific neuropsychological profile of children with FAS can currently be defined. Most of these children show below-average performance [19-27]. Functional abnormalities of the CNS should be evaluated by means of standardized neuropsychological tests together with behavioral assessment by a psychologist or physician [27]. Bell et al. found in a cohort from 2 FAS centers, that 5,9\% of children with FASD showed epilepsy. THhs is much higher than the $0,6 \%$ prevalence found in the normal population [28,29]. THere is no agreement in the literature regarding a recorded cut-off value for the presence of microcephaly in children with FAS.

Studies about the head circumference in children with FAS yielded conflicting results. Therefore, a head circumference <or= third percentile and a head circumference $<$ or $=10$ th percentile fulfill the criteria for the diagnostic category CNS abnormalities $[13,30]$ Owing to the limited evidence on structural abnormalities of the CNS such as volume reduction of the cerebellum and thickening of the cortex,it is agreed, that structural CNS abnormalities other than microcephaly, cannot currently be used as criteria for the diagnosis of FAS [31-36]

Importance of confirmation of maternal alcohol consumption in FAS: Burd et al. [37] investigated the importance of confirmation of alcohol consumption of the mother for the certainty of the diagnosis of FAS. In cases where maternal alcohol consumption could not be confirmed sensitivity for the diagnosis FAS was higher (unconfirmed 89\%,confirmed 85\%), while specificity was lower $(71,1 \%$ versus $82,4 \%)$. In other words,more children with FAS actually have FAS diagnosed,when alcohol consumption by their mother is not confirmed. Documentation of maternal alcohol intake is difficult. Many mothers are not questioned about their alcohol consumption during pregnancy, because carers are worried about loss of trust in the caregiving relationship. Otherwise mothers frequently deny alcohol use during pregnancy for reasons of social acceptability. The diagnosis of FAS remains difficult, because the characteristic abnormalities in children with FAS change with age. Facial abnormalities and growth deficiencies are obvious in childhood,but less distinct in adolescence and adulthood [38].

\section{ARND}

Unfortunately children with FAS represent only the tip of the iceberg of affected children, as numerous children exposed to alcohol in utero have significant physical or neuro developmental abnormalities, without all the features of FAS [39]. Alcohol related neurobehavior disorder (ANRD) refers to a constellation of neurobehavioral and central nervous system effects, occuring in the absence of the characteristic facial and growth abnormalities associated with FAS. These abnormalities include; head circumference $<$ or equal to the $10^{\text {th }}$ percentile,learning disabilities, poor impulse control, seizures, deficits in higher level receptive and expressive language, and problems with mathematical skills, memory, attention and judgement [40].

\section{ARBD}

Individuals that exhibit the typical FAS facies along with specific structural anomalies, that are known to be associated with alcohol exposure, such as low set ears,micrognathia, epicanthal folds, low nasal bridge, short upturned nose,strabismus, clinodactyly, "hockey stick" palmar crease, radioulnar synostosis, renal anomalies and cardiac defects, but have normal growth and development, are classified as having alcohol-related birth defects (ARBD)-[41]. The prevalence of ARND and ARBD is estimated to be at least 4 times more common than FAS $[42,43]$. Combining prevalence rates for FAS, ARND And ARBD indicates that $1 \%$ to $3 \%$ of all children born in the United States are affected by alcohol. This is probably an underestimation,because primary care providers and others, who care for children, do not routinely screen for FASD [44].

\section{Conclusion}

FASDs are still the leading cause of birth defects,intellectual and neurodevelopmental disabilities. There is a global need to establish an universal public health message about the potential harm of prenatal alcohol exposure and a routine screening protocol, according to the WHO $[8,10]$. Ten percent of pregnant women use alcohol and $1 \%$ to $3 \%$ of all children born in the United States are affected by alcohol.

\section{References}

1. Jones KL, Smith DW, Ulleland CN, Streissguth P (1973) Pattern of malformation in offspring of chronic alcoholic mothers. Lancet 1(7815): 1261-1271.

2. Riley EP, Infante MA, Warren KR (2011) Fetal alcohol spectrum disorders: an overview. Neuropsychol Rev 21(2): 73-80.

3. Surgeon General Advisory on Alcohol and Pregnancy: Surgeon General's advisory on alcohol and pregnancy (1981). FDA Drug Bull 11(2): 9-10.

4. US Surgeon General Releases Advisory on Alcohol Use in Pregnancy (2005).

5. Lange S, Probst C, Gmel G, Rehm J, Burd L et al. (2017) Global Prevalence of Fetal Alcohol Spectrum Disorder Among Children and Youth: A Systematic Review and Meta-analysis. JAMA Pediatr 171(10): 948-956.

6. Hoyme HE, May PA, Kalberg WO, Piyadasa K, Phillip GJ et al. (2005) A practical clinical approach to diagnosis of fetal alcohol spectrum disorders; clarification of the 1996 institute of medicine criteria. Pediatrics 115(1): 39-47. 
7. Manning MA, Hoyme HE (2007) Fetal Alcohol Spectrum Disorders: A practical clinical approach to diagnosis. Neurosci Biobeh Rev 31(2): 230-238.

8. Bertrand J, Floyd LL, Weber MK (2005) Fetal Alcohol Syndrome Prevention Team, Division of Birth Defects and Developmental Disabilities. National Center on Birth Defects and Developmental Disturbances. Centers for Disease Control and Prevention (CDC). Guidelines for identifying and refering persons with fetal alcohol syndrome. MMWR Recomm Rep 54(RR-11):1-14.

9. Williams JF, Smith VC (2015) Committee on Substance Abuse. Pediatrics 136(5): e1395-1406

10. Popova S, Lange S, Probst C (2017) Estimation of national,regional and global prevalence of alcohol use during pregnancy and fetal alcohol syndrome; a systematic review and meta-analysis. The Lancet Global Health 5(3):e290-e299.

11. Landgraf MN, Nothacker M, Kopp IB (2013) The Diagnosis of Fetal Alcohol Syndrome. Dtsch Ärztebl Int 110(42): 703-710.

12. Klug MG, Burd L, Martsolf JT (2003) Body mass index in fetal alcohol syndrome. Neurotoxicol Teratol 25(6): 689-696.

13. Day NL, Leech SL, Richardson GA, Cornelius MD, Robles N et al. (2002) Prenatal alcohol exposure predicts continued deficits in offspring size at 14 years of age. Alcohol Clin Exp Res 26(10): 1584-1591.

14. Jones KL, Smith DW, Hanson JW (1976) The fetalalcohol syndrome:clinical delineation. Ann N Y Acad Sci 273: 130-139.

15. Clarren SK, Sampson PD, Larsen J, Donnell DJ, Barr HM, et al. (1987) Facial effects of fetal alcohol exposure:assesssment by photographs and morphometric analysis. Am J Med Genet 26(3): 651-666.

16. Astley SJ, Clarren SK (1995) A fetal alcohol syndrome screening tool Alcohol Clin Exp Res 19(6): 1565-1571.

17. Astley SJ, Clarren SK (2000) Diagnosing the full spectrum of fetal alcohol exposed individuals: introducing the 4-digit diagnostic code. Alcohol Alcohol 35(4): 400-410.

18. Astley SJ (2004) FAS Diagnostic and Prevention Network, University of Washington: Diagnostic guide for fetal alcohol spectrum disorder: the 4- digit diagnostic code.

19. Mattson SN, Roesch SC, Fagerlund A, Jones KL, May PA, et al. (2010) Toward a neurobehavioral profile: fetal spectrum disorders. Alcohol Clin Exp Res 34(9): 1640-1650.

20. Astley SJ, OLson HC, Kerns K, Allison B, Elizabeth HA, et al. (2009) Neuropsychological and behavioral outcomes from a comprehensive magnetic resonance study of children with fetal alcohol spectrum disorders. Can J Clin Pharmacol 16(1): e178-e201.

21. Aragon AS, Coriale G, Fiorentino D, Kalberg WO, Buckley D, et al. (2008) Neuropsychological characteristics of Italian children with fetal alcohol spectrum disorders. Alcohol Clin Exp Res 32(11): 1909-1919.

22. Thorne JC, Coggins TA (2008) A diagnostically promising technique for tallying normal reference errors in the narratives of school-aged children with Foetal Alcohol Spectrum Disorders (FASD) Int J Lang Comm Disord 43(5): 570-594.

23. Vaurio L, Riley EP, Mattson SN (2011) Neuropsychological Comparison of Children with Heavy Prenatal Exposure and IQ-Matched Comparison Group. J Int Neuropsychol Soc 17(3): 463-473.

24. Pei J, Denys K, Hughes J, Rasmussen C (2011) Mental health issues in fetal alcohol spectrum disorder. J Ment Health 20(5): 438-448.

25. Rasmussen C, Benz J, Pei J, Andrew G, Schuller G, et al. (2010) The impact of teen ADHD co-morbidity on the diagnosis of FASD. Can J Clin Pharmacol 17(1): e165-e176.

26. Fagerlund A, Autti RI, Eugene H, Sarah NM, Marit K (2011) Risk factors for behavioral problems in foetal alcohol spectrum disorders. Acta Paediatr 100(11): 1481-1488.
27. Nash K, Koren G, Rover J (2011) A differential approach for examining the behavioural phenotype of fetal alcohol spectrum disorders. J Popul Ther Clin Pharmacol 18(3): e440-e453.

28. Bell SH, Stade B, Reynolds JN, Rasmussen C, Andrew G, et al. (2010) The remarkable high prevalence of epilepsy and seizure disorders in fetal alcohol spectrum disorders. Alcohol Clin Exp Res 34(6): 1084-1089.

29. Russ SA, Larson K, Halfon N (2012) A National profile of childhood epilepsy and seizure disorder. Pediatrics 129(2):256-64

30. Handmaker NS, Rayburn WF, Meng C, Bell JB, Rayburn BB, et al. (2006) Impact of alcohol exposure after pregnancy recognition on ultrasonographic fetal growth measures. Alcohol Clin Exp Res 30(5): 892-898.

31. Archibald SL, Fennema NC, Gamst A, Riley EP, Mattson SN, et al. (2001) Brain dysmorphology in individuals with severe prenatal alcohol exposure. Dev Med Child Neurol 43(3): 148-154

32. Geuze E, Vermetten E, Bremner JD (2005) MR-based in vivo hippocampal volumetrics: 2.Findings in neuropsychiatric disorders. Mol Psychiatry 10(2): 160-184.

33. Sowell ER, Mattson SN, Kan E, Thompson PM, Riley EP, et al. (2008) Abnormal cortical thickness and brain-behavior correlation patterns in individuals with heavy prenatal alcohol exposure. Cereb Cortex 18(1): 136-144.

34. Astley SJ, Aylward EH, OLson HC, Kerns K, Brooks A, et al. (2009) Magnetic resonance imaging outcomes from a comprehensive magnetic resonance study of children with fetal alcohol spectrum disorders. Alcohol Clin Exp Res 33(10): 1671-1689.

35. Bjorkquist OA, Fryer SL, Reiss AL, Mattson SN, Riley EP (2010) Cingulate gyrus morphology in children and adokescents with fetal alcohol spectrum disorders. Psychiatry Res 181(2): 101-107.

36. Yang Y, Rousotte F, Kan E, Sulik KK, Mattson SN, et al. (2011) Abnormal Cortical Thickness Alterations in Fetal Alcohol Spectrum Disorders and Their Relationships with Facial Dysmorphology. Cereb Cortex 22(5): 1170-1179.

37. Burd L, Klug MG, Li Q, Kerbeshian J, Martsolf JT (2010) Diagnosis of fetal alcohol spectrum disorders: a validity study of the fetal alcohol syndrome checklist. Alcohol 44(7-8): 605-614.

38. Spohr HL, Steinhauser (2008) TC Fetal alcohol spectrum disorders and their persisting sequelae in adult life. Dtsch Ärztebl Int 105(41): 693698.

39. Mattson SN, Riley EP, Gramling L, Delis DC, Jones KL (1997) Heavy prenatal alcohol exposure withor without physical features lead to IQ deficits. J Pediatr 131(5): 718-721.

40. National Institute on Alcohol Abuse and Alcoholism (NIAAA) Recognizing Alcohol Related Neurodevelopmental Disorder (ARND) in Primary Health Care of Children. Interagency Coordinating Committee on Fetal Alcohol Spectrum Disorders ( ICCFASD) 2011.

41. Hoyme HE, Karlberg WO, Elliot AJ, Jason Blankenship, David B, et al. (2016) Updated Clinical Guidelines for Diagnosing Fetal Alcohol Spectrum Disorders. Pediatrics 138(2): e20154256.

42. May PA, Gossage JP, White CM, Goodhart K, Decoteau S, et al. (2004) Alcohol consumption and other maternal risk factors for fetal alcohol syndrome among three distinct samples of women before, during and after pregnancy: the risk is relative. Am J Med Genet 127C(1): 10-20.

43. May PA, Gossage JP (2001) Estimating the prevalence of fetal alcohol syndrome. Alcohol Res Health 25(3): 159-167.

44. Graham JM, Hanson JW, Darby BL, Barr HM, Streissguth AP (1998) Independent dysmorphology evaluations at birth and 4 years of age for children exposed to varying amounts of alcohol in utero. Pediatrics 81(6): 772-778. 
(C) (P) This work is licensed under Creative Commons Attribution 4.0 License

DOI: 10.32474/IGWHC.2018.01.000104

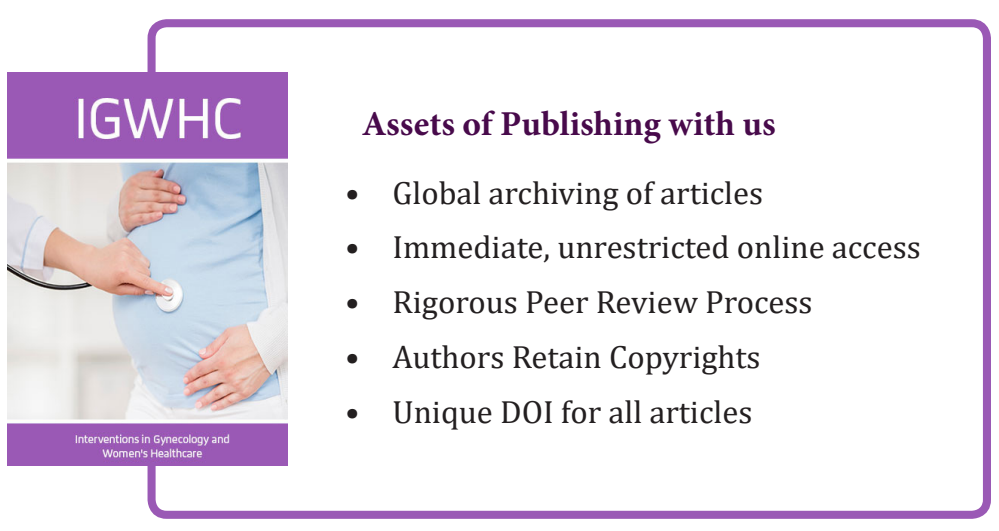

